\title{
Cortical and Subcortical Coordination of Visual Spatial Attention Revealed by Simultaneous EEG-fMRI Recording
}

\author{
기 Jessica J. Green, ${ }^{1,2}$ CCarsten N. Boehler, ${ }^{1,3}$ Kenneth C. Roberts, ${ }^{1}$ Ling-Chia Chen, ${ }^{1,5}$-Ruth M. Krebs, ${ }^{1,3}$ Allen W. Song, ${ }^{4}$ \\ and $\triangleright^{-M a r t y ~ G . ~ W o l d o r f f ~}{ }^{1,6}$ \\ ${ }^{1}$ Center for Cognitive Neuroscience, Duke University, Durham, North Carolina 27708, ${ }^{2}$ Department of Psychology, Institute for Mind and Brain, and McCausland \\ Center for Brain Imaging, University of South Carolina, Columbia, South Carolina 29201, ${ }^{3}$ Department of Experimental Psychology, Ghent University, Henri \\ Dunantlaan 2, 9000 Ghent, Belgium, ${ }^{4}$ Brain Imaging and Analysis Center, Duke University School of Medicine, Durham, North Carolina 27710, ${ }^{5}$ Neuropsychology \\ Laboratory, Department of Psychology, European Medical School, University of Oldenburg, Lower Saxony, Oldenburg 26129, Germany, and ${ }^{\circ}$ Department of \\ Psychiatry, Duke University, Durham, North Carolina 27708
}

Visual spatial attention has been studied in humans with both electroencephalography (EEG) and functional magnetic resonance imaging (fMRI) individually. However, due to the intrinsic limitations of each of these methods used alone, our understanding of the systemslevel mechanisms underlying attentional control remains limited. Here, we examined trial-to-trial covariations of concurrently recorded EEG and fMRI in a cued visual spatial attention task in humans, which allowed delineation of both the generators and modulators of the cue-triggered event-related oscillatory brain activity underlying attentional control function. The fMRI activity in visual cortical regions contralateral to the cued direction of attention covaried positively with occipital gamma-band EEG, consistent with activation of cortical regions representing attended locations in space. In contrast, fMRI activity in ipsilateral visual cortical regions covaried inversely with occipital alpha-band oscillations, consistent with attention-related suppression of the irrelevant hemispace. Moreover, the pulvinar nucleus of the thalamus covaried with both of these spatially specific, attention-related, oscillatory EEG modulations. Because the pulvinar's neuroanatomical geometry makes it unlikely to be a direct generator of the scalp-recorded EEG, these covariational patterns appear to reflect the pulvinar's role as a regulatory control structure, sending spatially specific signals to modulate visual cortex excitability proactively. Together, these combined EEG/fMRI results illuminate the dynamically interacting cortical and subcortical processes underlying spatial attention, providing important insight not realizable using either method alone.

Key words: attentional control; EEG; fMRI; pulvinar; visual cortex

Significance Statement

Noninvasive recordings of changes in the brain's blood flow using functional magnetic resonance imaging and electrical activity using electroencephalography in humans have individually shown that shifting attention to a location in space produces spatially specific changes in visual cortex activity in anticipation of a stimulus. The mechanisms controlling these attention-related modulations of sensory cortex, however, are poorly understood. Here, we recorded these two complementary measures of brain activity simultaneously and examined their trial-to-trial covariations to gain insight into these attentional control mechanisms. This multi-methodological approach revealed the attention-related coordination of visual cortex modulation by the subcortical pulvinar nucleus of the thalamus while also disentangling the mechanisms underlying the attentional enhancement of relevant stimulus input and those underlying the concurrent suppression of irrelevant input.

\section{Introduction}

A key function of spatial attention is to select for further scrutiny the most relevant parts of the vast sensory input that we experi-

Received Jan. 30, 2017; revised June 7, 2017; accepted July 5, 2017.

Author contributions: C.N.B. and M.G.W. designed research; J.J.G., C.N.B., K.C.R., L.-C.C., and R.M.K. performed research; A.W.S. contributed unpublished reagents/analytic tools; J.J.G. analyzed data; J.J.G., C.N.B., K.C.R., L.-C.C., R.M.K., A.W.S., and M.G.W. wrote the paper.

This work was supported by the National Institutes of Health (Grant R01-NS051048 to M.G.W. and Grant R24MH106048 to A.W.S.), the Natural Sciences and Engineering Research Council (Postdoctoral Fellowship to J.J.G.), and the German Research Foundation (Postdoctoral Fellowship B0 3345/1-1 to C.N.B.) ence continuously in life, thereby enhancing detection and discrimination of items occurring at an attended location (Posner, 1980). This enhanced processing is thought to rely on top-down signals from frontal and parietal cortex, which bias and, ulti-

The authors declare no competing financial interests.

Correspondence should be addressed to Jessica Green, Institute for Mind and Brain, University of South Carolina, 1800 Gervais St., Columbia, SC 29201. E-mail: jessica.green@sc.edu, or to Marty Woldorff, Center for Cognitive Neuroscience, Duke University, Box 90999, Durham, NC 27708, Email: woldorff@duke.edu.

DOI:10.1523/JNEUROSCI.0326-17.2017

Copyright $\odot 2017$ the authors $\quad 0270-6474 / 17 / 377803-08 \$ 15.00 / 0$ 
mately, amplify sensory processing of stimuli presented at the attended location relative to those at unattended locations (Corbetta and Shulman, 2002). To understand the neural mechanisms underlying spatial attention, human electrophysiology and neuroimaging have largely focused on cortical regions; however, animal electrophysiology, clinical observations, and theoretical models have implicated the involvement of subcortical areas such as the superior colliculus and the pulvinar nucleus of the thalamus in the orienting of visual spatial attention (Petersen et al., 1987; LaBerge and Buchsbaum, 1990; Karnath et al., 2002; Shipp, 2004; Saalmann and Kastner, 2011).

Here, we examined the cortical and subcortical mechanisms of anticipatory modulation of sensory cortex during voluntarily directed spatial attention. During cue-triggered visuospatial shifts of attention, spatially specific modulations are observed in occipital cortex; in particular, the effects in the two hemispheres depend on where in space one is attending. Studies using functional magnetic resonance imaging (fMRI) have consistently shown relative blood-oxygenation-level-dependent (BOLD) signal increases in visual cortex contralateral to the direction of attention, interpreted as the enhancement of processing for the attended location of space. Likewise, event-related potentials (ERPs) (Harter et al., 1989; Hopf and Mangun, 2000) and eventrelated gamma-band $(>30 \mathrm{~Hz})$ electroencephalography (EEG) oscillations (Ward, 2003; Jensen et al., 2007; Doesburg et al., 2008) over occipital scalp have been linked to anticipatory enhancement in the contralateral visual cortex. In contrast, alphaband EEG $(\sim 8-12 \mathrm{~Hz})$ has a negative relationship with the BOLD signal, with increased alpha being linked to decreased BOLD activity and decreased cortical excitability (Goldman et al., 2002; Laufs et al., 2003; Scheeringa et al., 2012). Although earlier studies suggested that alpha-power decreases contralateral to the attended location lead to enhanced processing at that location (Yamagishi et al., 2005), subsequent studies have linked the underlying hemispheric asymmetry to alpha-power increases contralateral to the to-be-ignored location of space (Kelly et al., 2006; Rihs et al., 2007), supporting a role of alpha oscillations in relative suppression of irrelevant information in the environment.

Although it is clear that attending to a location in space produces spatially specific modulations of activity in visual cortex, it remains unclear whether the effects observed with fMRI and those observed with EEG reflect the same underlying neural mechanisms. Moreover, activity in subcortical structures is generally inaccessible with scalp EEG due to their neuroanatomical structure (Nunez and Srinivasan, 2006). The modulatory effects of subcortical structures on cortical EEG, however, can be ascertained when EEG is recorded concurrently with fMRI (Huster et al., 2012) by examining the trial-to-trial covariations of the activity measured with the two methods. Here, we recorded EEG and fMRI simultaneously during a cued spatial attention task to link these EEG and fMRI modulations.

On each trial, a centrally presented directional cue predicted where a pair of to-be-discriminated target stimuli would appear (Fig. 1). Periods of cue-elicited spatially specific modulations of occipital scalp EEG were identified and extracted in the alphafrequency $(8-12 \mathrm{~Hz})$ and gamma-frequency $(38-42 \mathrm{~Hz})$ bands on each trial. These single-trial amplitudes were then analyzed for their covariations with the fMRI responses for the corresponding trials, revealing coordinated interactions between cortical and subcortical brain regions for the control of visual spatial attention.

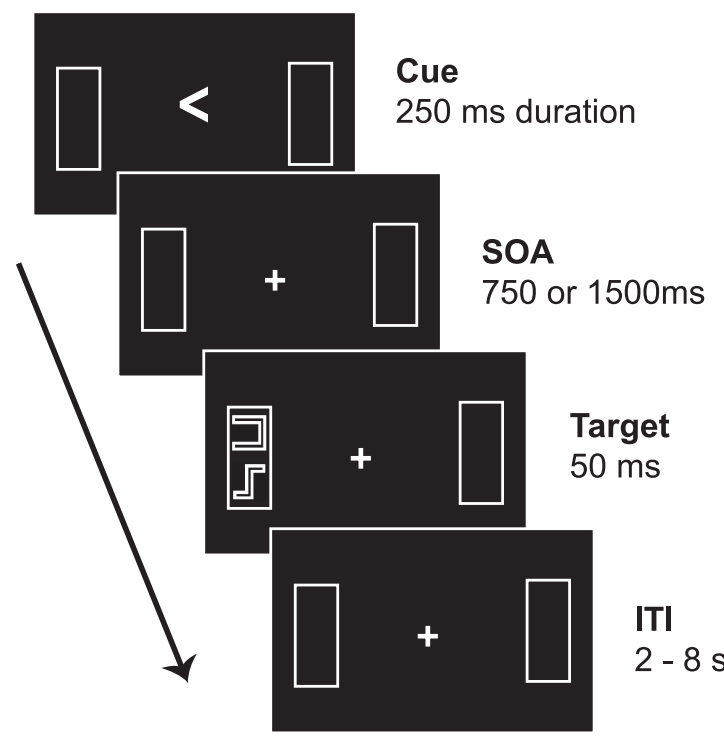

Figure 1. Schematic diagram of a target present trial (see text for details)

\section{Materials and Methods}

Participants. Twenty-five participants took part in the study after providing informed consent. Data from eight participants were unusable due to excessive movement artifacts in the fMRI (three participants) or ocular artifacts in the EEG (five participants). Data from the remaining 17 participants ( 12 female, mean age $=24.4 \pm 3.5$ years, all right handed with normal or corrected-to-normal vision) were used for analysis. All experimental protocols were approved by the Duke University Institutional Review Board.

Stimuli and paradigm. Throughout the task, a small fixation spot remained on the screen, along with rectangular box outlines in the left and right visual fields $\sim 7^{\circ}$ from fixation that served as attentional landmarks within which the target stimuli could be presented (Fig. 1). Participants were instructed to maintain fixation, and eye movements were monitored to ensure proper fixation. Each trial started with an arrow cue presented at fixation for $250 \mathrm{~ms}$. Left and right arrows served as attention-directing cues (attend cues) and were 100\% predictive of the potential target location. On two-thirds of attend-cue trials, a target display was presented for $50 \mathrm{~ms}$ at a stimulus onset asynchrony (SOA) of either $750 \mathrm{~ms}$ (short-SOA) or $1500 \mathrm{~ms}$ (long-SOA) after cue onset. On one-third of trials, no target display was presented (cue-only condition; Woldorff et al., 2004). Target displays consisted of two shapes selected from a stimulus set of five shapes (box outlines of a " + ," a "C," a " $Z$," and a mirror-reversed "C" and "Z"; see Boehler et al., 2011) presented within the cued landmark box. Participants indicated whether the two shapes were identical ( $50 \%$ of target trials) or different ( $50 \%$ of target trials) by pressing a button with the right index or middle finger, respectively. An upward-pointing arrow cue indicated that no target would appear on that trial (interpret-cue trial), thus serving as a control cue requiring the same sensory processing and meaning-interpretation as the attend cues but not engaging attentional orienting.

A total of 360 attend cue trials (60 for each of short-SOA, long-SOA, and cue-only for each of left and right cues) and 132 interpret cue trials were presented. These trials were separated into three functional runs of $15 \mathrm{~min}$ each, with three $10 \mathrm{~s}$ breaks within each run. The sequence of conditions was varied pseudorandomly with discrete intertrial intervals that varied among 4,6 , and $8 \mathrm{~s}$, with a mean of $5 \mathrm{~s}$. An additional $0-200 \mathrm{~ms}$ jitter was added to each trial to decorrelate the cue onsets from the onsets of the fMRI volume acquisitions.

fMRI acquisition and preprocessing. MRI data were acquired on a 3 tesla GE MR750 system. A 3D spoiled-GRE sequence (EFGRE3D) without inversion recovery preparation was used to acquire structural T1 images $(0.9375 \mathrm{~mm}$ in-plane resolution, $1.2 \mathrm{~mm}$ slice thickness; field of view: $24 \mathrm{~cm} * 24 \mathrm{~cm}^{*} 20 \mathrm{~cm} ; \mathrm{TR}=5.848, \mathrm{TE}=1.932$, flip angle $\left.=12^{\circ}\right)$ for each 
participant. Functional images were acquired with a customized inward spiral imaging sequence $\left(\mathrm{TR}=2000 \mathrm{~ms} ; \mathrm{TE}=30 \mathrm{~ms}\right.$; flip angle $=60^{\circ}$; slew rate $=120$; 40 slices with $3 \times 3 \times 3 \mathrm{~mm}$ resolution; AC-PC orientation) that was modified to prevent saturation of the EEG amplifier during simultaneous recording. Each run consisted of 456 functional images, with the first five discarded to allow for reaching steady-state magnetization. No task was presented during the last eight volumes to fully sample the hemodynamic response to the last event.

All image preprocessing and analysis was performed using SPM8 (RRID: SCR_007037). Functional images were slice-time corrected, spatially realigned, and spatially normalized to the SPM template using the coregistered individual T1 images. Functional images were then resampled to a voxel size of $2 \times 2 \times 2 \mathrm{~mm}$ and smoothed with a $6 \mathrm{~mm}$ full-width half-maximum Gaussian kernel.

Concurrent EEG acquisition and preprocessing. EEG data were acquired from 64 electrodes set in a custom electrode cap with extended scalp coverage (Woldorff et al., 2002), including 62 scalp recording sites, an electrode under the left eye for monitoring blinks and vertical eye movements, and an electrode on the upper back for recording the electrocardiogram (BrainAmp MR Plus, Brain Products, RRID: SCR_009443). Horizontal eye movements were detected using electrodes lateral to the left and right eyes. All signals were recorded with a band pass of $0.016-$ $250 \mathrm{~Hz}$ and digitized at $5000 \mathrm{~Hz}$, referenced during recording to scalp site $\mathrm{Cz}$. Electrode impedances were lowered to $<5 k \Omega$ before recording.

Removal of the MR gradient artifact, as well as heartbeat detection for ballistocardiogram (BCG) correction, were performed in Brain Vision Analyzer 2 (Brain Products, RRID: SCR_009443). MR gradient artifacts were removed using an average template subtraction method (Allen et al., 2000). An fMRI slice-based template of the artifact was created from a sliding average of $19150 \mathrm{~ms}$ epochs, which was then subtracted from the EEG segment time locked to each slice acquisition. The EEG was then low-pass filtered to $100 \mathrm{~Hz}$ and downsampled to $500 \mathrm{~Hz}$. Heartbeat detection using the ECG channel was then performed using a semiautomatic template-matching procedure. The resulting markers were reviewed manually and adjusted and the EEG exported for subsequent analysis in MATLAB (The MathWorks, RRID: SCR_001622). Removal of the BCG artifact was performed using the Optimal Basis Set procedure implemented in the FMRIB plugin for EEGLAB (Niazy et al., 2005) using the top four principal components for correction.

The artifact-corrected EEG was then segmented into $3.5 \mathrm{~s}$ epochs $(1 \mathrm{~s}$ prestimulus to $2.5 \mathrm{~s}$ poststimulus) time locked to cue onsets and inspected manually to detect any trials containing blinks, eye movements, or excessive noise. All participants used in the analysis had at least $70 \%$ of trials retained after artifact rejection. Data were then digitally rereferenced to the average of all scalp channels.

EEG-fMRI covariation. Because low-amplitude ERP components can be difficult to detect in single-trial data (Jung et al., 2001), we focused specifically on time-frequency effects to improve signal-to-noise in our single-trial measurements and home in on the attentional suppression and enhancement processes previously associated with the alpha and gamma bands, respectively. EEG epochs were transformed into the timefrequency domain in EEGLAB (RRID: SCR_007292) using a fast Fourier transform approach with Hanning window tapering. Because we were interested in how the change in alpha and gamma power on each trial related to changes in the fMRI BOLD signal, single-trial data were normalized using the $200 \mathrm{~ms}$ prestimulus baseline period for each epoch. Mean event-related spectral perturbations across all participants were calculated for occipital electrodes (TO1/2, $\mathrm{P} 3 \mathrm{i} / 4 \mathrm{i}, \mathrm{O}^{\prime} / 2^{\prime}$, $\mathrm{PO} 1 / 2$ ) ipsilateral and contralateral to the to-be-attended direction in two frequency ranges of interest, alpha $(8-12 \mathrm{~Hz})$ and gamma $(38-42 \mathrm{~Hz})$, and used to identify time windows of interest for further analysis. However, averaging of individually baselined epochs of power, which are necessarily positive, produces an overall positive shift in mean power (Grandchamp and Delorme, 2011). Although this positive shift changes the overall morphology of the average event-related spectral perturbation (ERSP) waveform, it does not affect the timing or frequency of the significant contralateral versus ipsilateral differences, which were our main focus here. For display purposes and to facilitate comparison with previous studies (Yamagishi et al., 2005; Kelly et al., 2006; Rihs et al., 2007), we also calculated mean ERSP responses
Table 1. Locations of functional ROls

\begin{tabular}{|c|c|c|c|c|}
\hline \multirow[b]{2}{*}{ Region } & \multicolumn{3}{|c|}{ Peak voxel location (MNI) } & \multirow{2}{*}{$\begin{array}{l}\text { Cluster size } \\
\text { (voxels) }\end{array}$} \\
\hline & $x$ & $y$ & $z$ & \\
\hline \multicolumn{5}{|c|}{ BOLD: attend cue - interpret cue } \\
\hline Left occipital & -42 & -60 & -8 & 493 \\
\hline Right occipital & 34 & -66 & -12 & 520 \\
\hline Left parietal & -24 & -58 & 46 & 282 \\
\hline Right parietal & 26 & -54 & 52 & 108 \\
\hline \multicolumn{5}{|c|}{ Covariation: ipsilateral alpha } \\
\hline Left occipital & -26 & -86 & 0 & 128 \\
\hline Right occipital & 38 & -80 & -4 & 290 \\
\hline \multicolumn{5}{|c|}{ Covariation: early contralateral gamma } \\
\hline Left pulvinar & -14 & -30 & 4 & 60 \\
\hline Right pulvinar & 14 & -34 & 6 & 79 \\
\hline
\end{tabular}

with baseline correction performed after averaging across trials (see Fig. 3). Three time-frequency windows were chosen for covariational analysis because they showed significant $(p<0.05)$ contralateral versus ipsilateral differences in occipital scalp activity: $800-1200 \mathrm{~ms}$ postcue for the alpha band and 400-600 ms and 1200-1400 ms for the gamma band.

The single-trial values were then extracted for each time-frequency window for both ipsilateral and contralateral electrode sites and included as parametric modulators in the SPM regression analyses. For each cueing condition (right short-SOA, long-SOA, and cue-only; left short-SOA, long-SOA, and cue-only; interpret cues), fMRI BOLD responses were modeled by a canonical hemodynamic response function with temporal and dispersion derivatives. Parametric modulators were included for right and left long-SOA and cue-only trials. The short-SOA trials were included in the design to encourage participants to shift attention to the cued location as quickly as possible, but were not included in the covariation analyses. Rest breaks were also modeled as regressors of no interest. These regressors were then entered into a general linear model (GLM) along with the six realignment parameters for each run.

Separate GLMs were constructed to model covariation for each timefrequency component (contralateral and ipsilateral for early gamma, alpha, and late gamma). For each analysis, we created contrasts to examine covariation for leftward and rightward shifts of attention separately to evaluate activity that varied with the direction of attention, as well as collapsed across leftward and rightward shifts to evaluate activity common to all attentional shifts. The analyses used a voxelwise threshold of $p<0.001$ (uncorrected), with an extent threshold of $k>25$ contiguous voxels. We also examined the results of the combined analyses using a smaller extent threshold of $k>10$ due to the possibility of observing nonlateralized activations in the small thalamic and midbrain structures that would not be visible with larger extent thresholds, but no additional subcortical activations were identified with this lower threshold.

Attention control regions were identified by contrasting attend cue versus interpret cue BOLD activity. Spatially specific activation of occipital cortex was assessed by contrasting BOLD activity on left cue attend trials with that on right cue attend trials (long-SOA and cue-only). For BOLD-only contrasts, a threshold of $p<0.01$ (FDR corrected) and an extent threshold of $k>25$ were applied. Significant clusters of activity from the attend cue versus interpret cue contrast were used to create functional ROIs in occipital and parietal cortex using MarsBaR (Brett et al., 2002; RRID: SCR_009605; Table 1).

\section{Results}

Standard fMRI analysis confirmed greater activation of the typical attention network in response to attend cues relative to interpret cues (Fig. 2), including increased BOLD signal in occipital and parietal cortices, inferior and superior frontal cortex, anterior insula, and the thalamus. Contrasts of responses to the attend left and attend right cues yielded expected relative increases in BOLD signal in the visual cortex contralateral to the direction of attention.

ERSP values for the alpha $(8-12 \mathrm{~Hz})$ and gamma $(38-42 \mathrm{~Hz})$ activity time locked to the cue onsets were extracted from four 


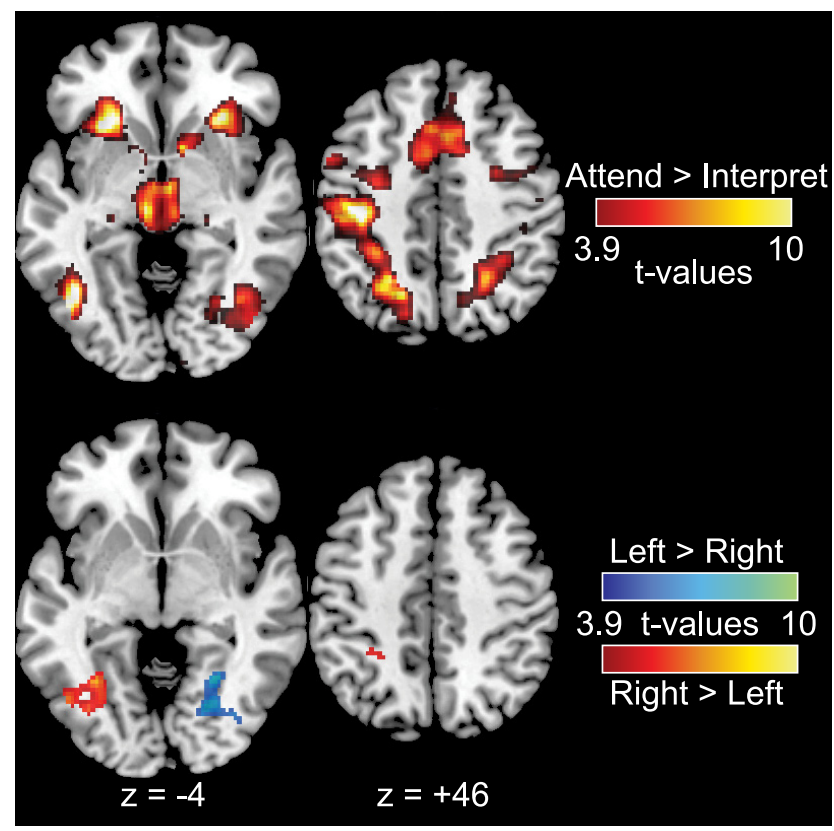

Figure 2. Average BOLD signal ( $p<0.01, \mathrm{FDR}$ corrected, $k=25$ ). Attentional control regions identified from the attend cue versus interpret cue BOLD contrast (top row) and spatially specific occipital activations identified from contrasting activity for leftward-directing versus rightward-directing cues.

posterior scalp electrodes over each hemisphere. A comparison between electrodes ipsilateral and contralateral to the cued direction was used to identify time windows of the oscillatory EEG for covariation analyses with the BOLD signal. Alpha power was greater ipsilateral to the cued direction beginning $\sim 600 \mathrm{~ms}$ after cue onset and lasting until the end of the cue period (i.e., until target onset on trials with long cue-target intervals; Fig. 3). Gamma power was greater contralateral to the cued direction in two time intervals: $400-600 \mathrm{~ms}$ and 1200-1400 ms (Fig. 3). Therefore, for covariation analysis, we selected three nonoverlapping time-frequency periods: Early gamma (38-42 hz; 400-600 ms), alpha (8-12 hz; 800-1200 ms), and late gamma $(38-42 \mathrm{~Hz}$; $1200-1400 \mathrm{~ms}$ ). Single-trial power values in these windows contralateral and ipsilateral to the direction of attention were then included as parametric modulators in the GLM analyses to identify voxels in the brain where the $\mathrm{MRI}$ BOLD signal covaried with the EEG power on a trial-to-trial basis.

\section{Pulvinar modulates attention-related changes in occipital EEG}

In the early time interval (400-600 ms after cue onset), gamma activity over occipital scalp contralateral to the direction of attention showed a positive covariation with BOLD signal in low-level visual cortex, left insula, and bilateral pulvinar (Fig. 4) regardless of the direction of attention. In contrast to the clear contralateral versus ipsilateral differences in the early gamma-band occipital EEG on the scalp (Fig. 3), no significant lateralizations for the early gamma-band/fMRI covariations with respect to the direction of attention were seen in occipital cortex.

The covariation observed in the pulvinar was particularly interesting because this subcortical structure, due to its anatomical geometry, does not produce an open field that can be picked up at the scalp, thereby making it inaccessible to direct EEG measures (Nunez and Srinivasan, 2006). Therefore, the observed covariation in the pulvinar likely results from it modulating cortical

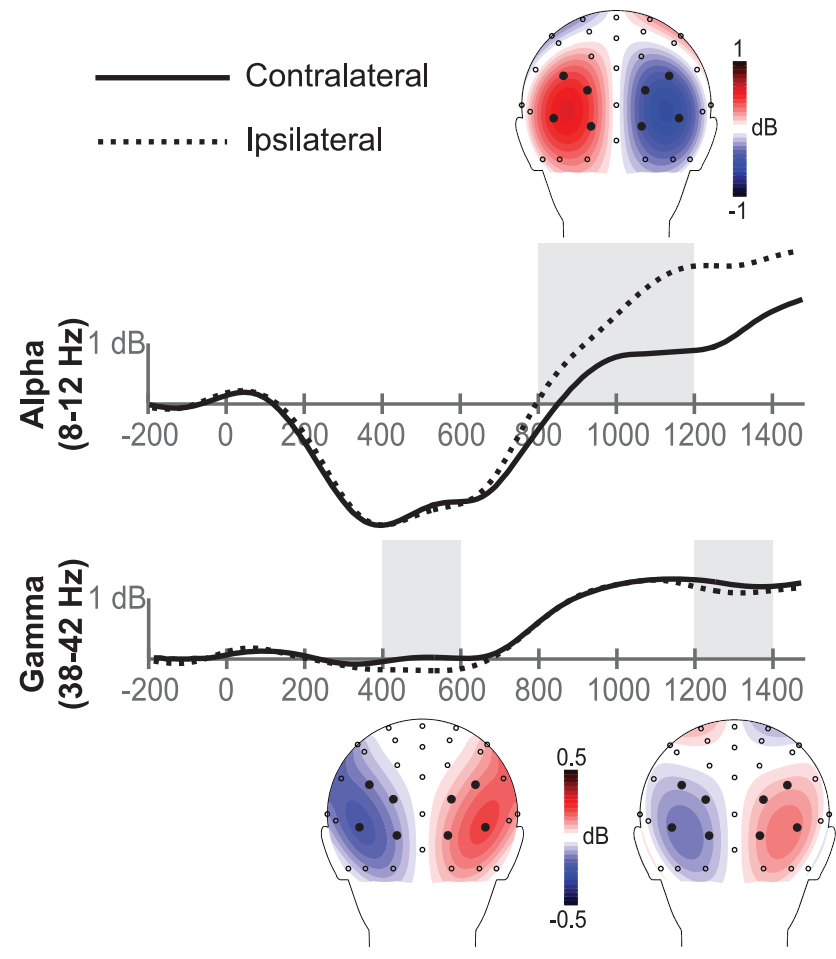

Figure 3. Average event-related responses and scalp topographies of contralateral-minus ipsilateral differences for alpha-band (top) and gamma-band (bottom) EEG. Light gray boxes denote time windows with significant $(p<0.05)$ differences between ipsilateral and contralateral electrodes that were used for covariational analyses.

activity that is then picked up by EEG. The pulvinar has been previously implicated in shifting spatial attention (Petersen et al., 1987; LaBerge and Buchsbaum, 1990) and the suppression of irrelevant information (Strumpf et al., 2013). Moreover, the pulvinar has been linked to the regulation of alpha oscillations and alpha-gamma cross-frequency coupling in visual cortex in monkeys (Saalmann et al., 2012) and to the generation of restingstate occipital alpha oscillations in human EEG-fMRI studies (Liu et al., 2012). However, given the lack of a direct scalp-level activity measure, the attentional modulation of occipital alpha is often attributed to frontal and parietal cortical structures without reference to the thalamus at all (Corbetta and Shulman, 2002; but see Shipp, 2004).

To further examine the role of the pulvinar in attentional control, we created functional ROIs for the left and right pulvinar derived from the early latency gamma covariation results (Fig. 4) and examined the alpha and late-gamma covariations within these ROIs. Unlike early gamma, in which covariation was present bilaterally in the pulvinar for both left and right cues, significant cue direction $X$ hemisphere interactions were found for both ipsilateral alpha $\left(F_{(1,16)}=7.44, p=0.015\right)$ and late contralateral gamma $\left(F_{(1,16)}=4.51, p=0.05\right)$ covariations (main effects of cue direction and hemisphere were all nonsignificant, $p>0.49)$. These alpha and late-gamma covariation effects, however, were in opposite hemispheres. Alpha power over occipital scalp ipsilateral to the direction of attention covaried positively with the BOLD signal in the ipsilateral pulvinar, whereas gamma power over contralateral occipital scalp covaried positively with the BOLD signal in the contralateral pulvinar. Notably, EEG from the opposite scalp sites (contralateral scalp for alpha and ipsilateral scalp for gamma) showed no significant covariation in the pulvinar $(p>0.63)$. 


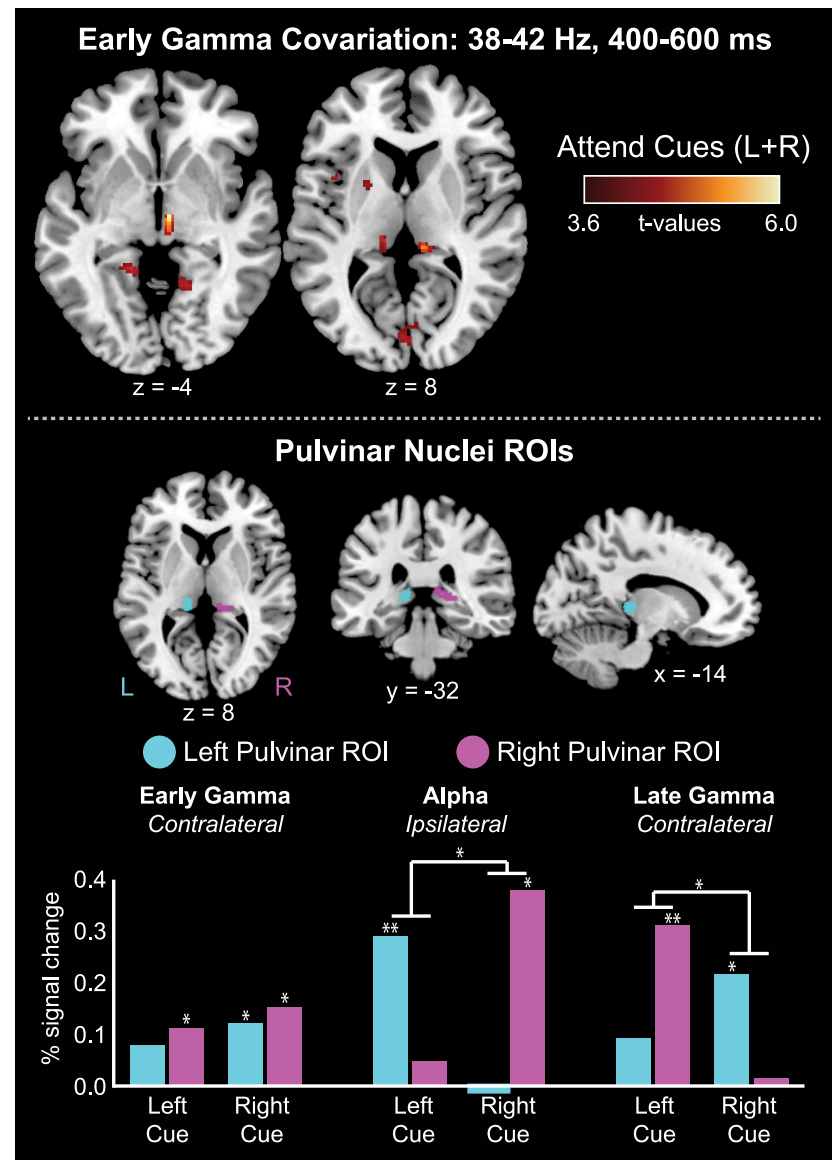

Figure 4. Covariations in the pulvinar. Early gamma for attend cues (left and right) positively covaried with BOLD signal in the pulvinar and early visual cortex ( $p<0.001$ uncorrected, $k>25$; top). This contrast was then used to create functional ROIs in the left and right pulvinar (bottom). Both ipsilateral alpha and late contralateral gamma showed spatially specific positive covariations in the pulvinar ROls (significant cue direction $\times$ hemisphere interaction). Early gamma covariations are shown only for comparative purposes because these regions were originally defined by having significant early gamma covariation activity. ${ }^{*} p<0.05$; ${ }^{* *} p<$ 0.01 .

Alpha and gamma reflect distinct mechanisms for anticipatory modulation of visual cortical activity

Alpha and late-gamma activity covaried with BOLD signal in occipital cortex, but the lateralization with respect to the direction of attention was reversed for the two frequency bands (Fig. 5). Ipsilateral increases in alpha power on the scalp were related to decreased BOLD signals in ipsilateral visual and parietal cortices. Conversely, contralateral increases in late gamma power on the scalp were related to increased BOLD signals in contralateral visual cortex. Neither contralateral alpha nor ipsilateral gamma significantly covaried with BOLD responses.

These alpha and gamma covariations also appeared to occur in distinct regions of visual cortex, with the alpha covariations occurring in more medial and posterior regions of occipital cortex and the gamma covariations in more lateral and anterior regions. This pattern suggests a hierarchical distinction in attentional suppression versus enhancement. For example, suppression of irrelevant information could take place at lower levels of processing, reducing processing of information from the to-beignored visual field earlier in the processing stream, whereas efficient target enhancement could happen at higher levels of visual processing specific to the relevant features of the target (Slagter et al., 2016).

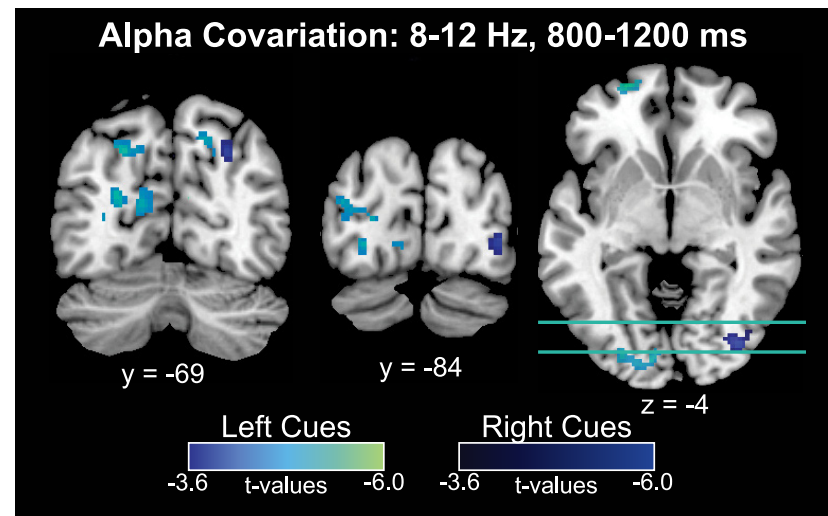

Late Gamma Covariation: 38-42 Hz, 1200-1400 ms

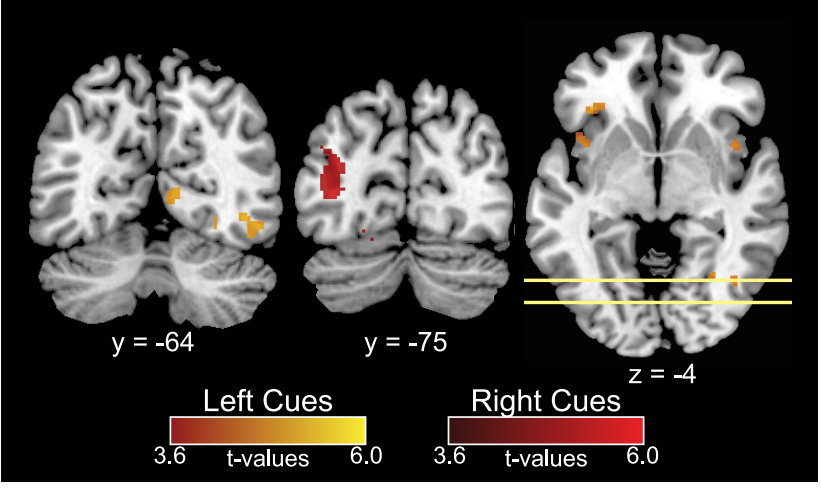

Figure 5. Covariation between BOLD and ipsilateral alpha (top) and contralateral late gamma (bottom). Alpha was negatively correlated with occipital and parietal BOLD activity in the hemisphere ipsilateral to the cued location, whereas late gamma was positively correlated with occipital BOLD signal contralateral to the cued location. $p<0.001$ uncorrected, $k=25$.

It is possible, however, that BOLD-EEG covariation in the occipital lobes actually does occur in the same locations for both the alpha and gamma frequencies, but appears distinct in the whole-brain analyses due to individual variability, along with sensitivity limitations in such analyses. Therefore, we examined covariational activity for early contralateral gamma, ipsilateral alpha, and late contralateral gamma within functional ROIs constructed from significant clusters observed in the alpha covariation analysis and in occipital and parietal ROIs delineated in the attend cue versus interpret cue fMRI BOLD contrast (Fig. 6). If the distinct alpha and gamma covariations are the result of poor sensitivity in the whole-brain analysis, then spatially specific covariations should be observed for both the alpha and late gamma within the two sets of occipital ROIs. In contrast, if alpha and gamma activity recorded at the scalp indeed reflects the targeting of distinct visual areas for attentional suppression versus for attentional enhancement, respectively, then spatially specific alpha and gamma covariations should occur in distinct ROIs.

As can be seen in Figure 6, no spatially specific activity (i.e., no cue direction $\times$ hemisphere interaction) was apparent for either the early or late gamma covariation within the alpha-derived ROIs. Within the occipital BOLD ROIs, however, spatially specific activity was observed, but only for the late contralateral gamma covariation. Within the parietal BOLD ROIs, significant covariations with early gamma were observed in both hemispheres for both left and right cues, but the activity was not spatially specific, mirroring the spatially nonspecific activity observed in the pulvinar during this same time-frequency window. Together, these results demonstrate that spatially specific attentional modulations in the 

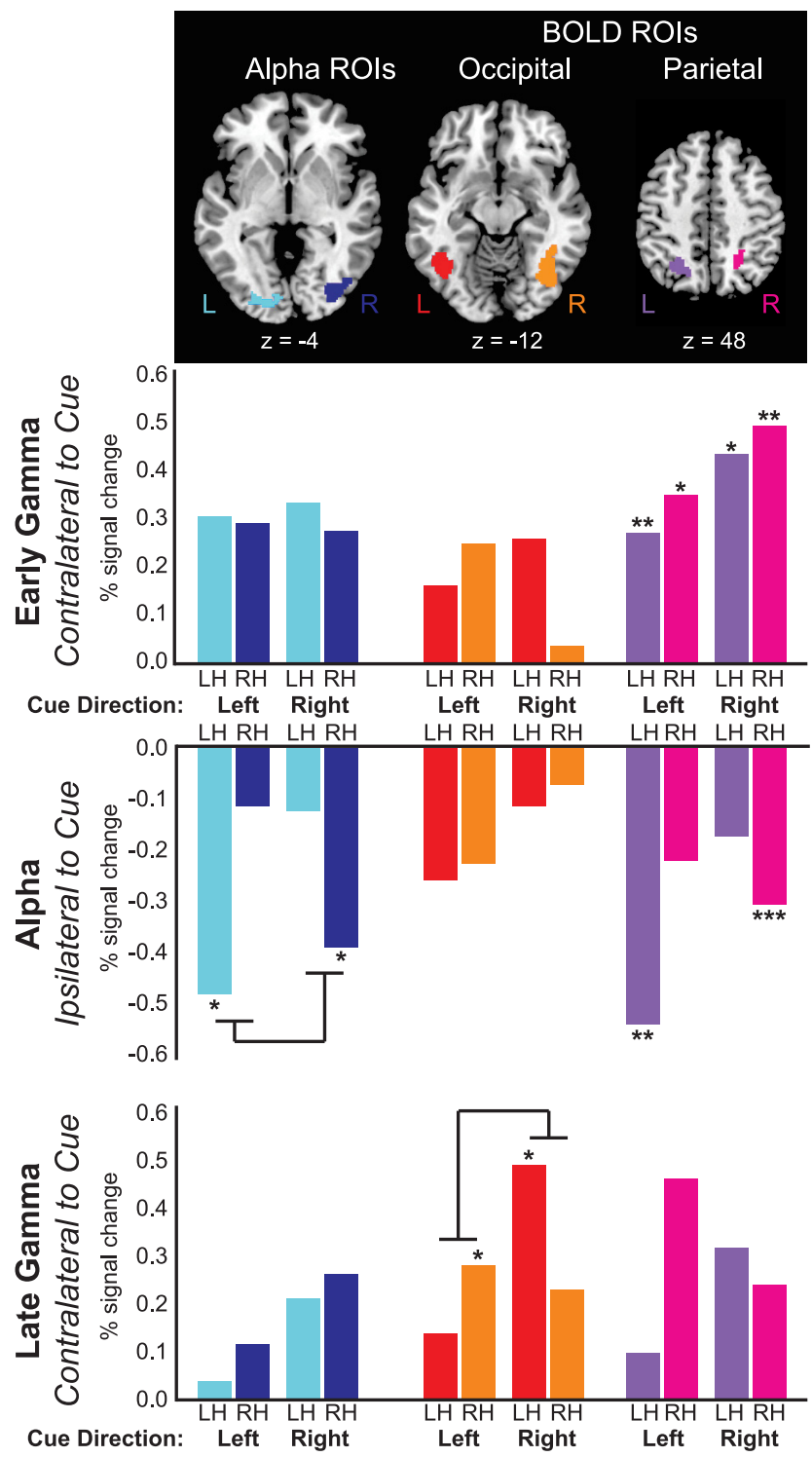

Figure 6. Covariations within functional ROls based on the alpha covariation (cyan and blue bars) and occipital (red and orange bars) and parietal (purple and magenta bars) BOLD responses. Spatially specific alpha and late-gamma covariations (significant hemisphere $\times$ cue direction interaction) occurred in distinct regions of visual cortex. Alpha covariations within the alpha-defined ROIs are shown for comparative purposes only because these regions were defined by having significant alpha covariation activity. Some evidence of spatially specific covariations was also seen in the parietal regions for alpha and late gamma, but these interactions did not reach significance. ${ }^{*} p<0.05 ;{ }^{* *} p<0.01 ;{ }^{* * *} p<0.001$.

alpha and late-gamma EEG are related to distinct BOLD modulations in different areas of visual cortex. More specifically, scalpmeasured changes in contralateral gamma are most closely related to the increased BOLD observed contralateral to the tobe-attended location in space. Conversely, alpha shows an inverse relationship with BOLD signal in early visual cortex ipsilateral to the to-be-attended location in space, providing further evidence that increases in alpha specifically reflect a mechanism for suppressing neural activity for to-be-ignored locations.

\section{Discussion}

For both the alpha-band and late-gamma-band EEG observed on the scalp, spatially specific modulations of activity in anticipation of an upcoming stimulus were related to modulations of the BOLD signal in the pulvinar and occipital cortex.

The fact that the pulvinar does not have the neuroanatomical geometry to be a primary generator of the scalp EEG activity itself (Nunez and Srinivasan, 2006) suggests that the covariational activity pattern in this subcortical structure reflects its proposed role as a regulatory control structure, sending spatially specific signals to modulate the excitability of visual cortex. It is possible that these covariations arise due to the pulvinar maintaining a condensed representation of visual cortex activity (Shipp, 2004). However, given recent evidence that the pulvinar is involved in filtering distracting information (Fischer and Whitney, 2012; Strumpf et al., 2013) and regulates cortical alpha oscillations as well as alpha-gamma cross-frequency coupling in visual cortex (Saalmann et al., 2012), we suggest it is more likely that the pulvinar is involved in directly modulating visual cortical activity.

The covariations in the pulvinar were all positive, but occurred in opposite hemispheres relative to the direction of attention for the different frequency bands. Increased alpha over ipsilateral occipital scalp was linked to increased fMRI activity in the ipsilateral pulvinar and to decreased fMRI activity in ipsilateral occipital cortex. Such a pattern is consistent with the hypothesis that increased alpha indexes suppression of activity in the hemisphere that processes the to-be-ignored region of space and that this suppression is orchestrated, at least in part, by thalamocortical connections from the pulvinar (Saalmann et al., 2012). In contrast, in the hemisphere processing the to-be-attended location, increased gamma late in the cue target interval was linked to increased fMRI activity in the contralateral pulvinar and occipital cortex, suggesting that the thalamocortical connections between the pulvinar and occipital cortex can both suppress and enhance processing.

One question that arises from these results, however, is why activity in the pulvinar is rarely observed in fMRI studies of spatial orienting despite some early PET (LaBerge and Buchsbaum, 1990) and animal (Petersen et al., 1987; Saalmann et al., 2012) studies suggesting that the pulvinar plays a functional role in shifting attention. Many fMRI studies of voluntary spatial attention have not reported thalamic activity in response to attentiondirecting cues, focusing instead on activations observed in attentional control regions in the frontal and parietal cortices, and for those that have reported thalamic activity, the attentional modulations were either not limited to the pulvinar part of the thalamus or did not show any spatial specificity (Kastner et al., 2004; Woldorff et al., 2004). As a result, anticipatory modulation of visual cortex is typically attributed to direct cortical input from these frontal and parietal regions (Liu et al., 2016; Marshall et al., 2015), whereas the pulvinar tends not to be included in many of the most commonly cited models of attention control (Corbetta and Shulman, 2002; Capotosto et al., 2009).

Our results provide evidence that the pulvinar does in fact play a key role in the shifting of spatial attention, while also providing possible explanations for why this activity is typically not observed in traditional fMRI studies. First, the covariations with alpha occurred in the pulvinar ipsilateral to the direction of attention, whereas covariations with gamma occurred in the contralateral pulvinar, with both of these cortical EEG measures covarying with increases in the pulvinar BOLD signal. Without the ability to separate the signals in the two hemispheres based on EEG frequency, any pulvinar activity would thus appear to be bilateral for both leftward- and rightward-directing cues, rather than being spatially specific (Kastner et al., 2004). Second, the covariations between the EEG and the pulvinar BOLD were time 
and frequency limited, as evidenced by the lack of spatially specific covariations in the early gamma time window. Activity in the pulvinar may be too short-lived to produce a strong event-related BOLD signal, or activity related to different time-frequency effects could cancel each other out in the slow event-related fMRI response. Finally, it is possible that the BOLD activity in the pulvinar varies from trial-to-trial with respect to attention, but that this variation simply averages out to a near-zero (or near baseline) value and thus is not observed in the average BOLD signal difference between conditions.

These considerations point to the value of combining multiple methods to investigate the role of thalamocortical communication in controlling attention. fMRI alone has not heretofore been able to delineate the contributions of the pulvinar in this type of top-down attentional control, and scalp-recorded EEG cannot be used to measure neural activity directly in this subcortical structure (Nunez and Srinivasan, 2006). By recording the EEG concurrently with fMRI, we were able to implicate the pulvinar as a control structure involved in the modulation of spatially specific occipital oscillatory EEG and cortical activation observed during visuospatial shifts of attention, presumably in conjunction with the attentional control regions in the frontal and parietal cortices.

A second important finding from this covariational analysis is that the attentional suppression (alpha) and enhancement (gamma) effects were observed in distinct regions of visual cortex, rather than simply in homologous regions of the two hemispheres. Positive covariations between BOLD signal and occipital gamma-band EEG most closely corresponded to the task-related changes in the average BOLD signal observed with PMRI alone, consistent with previous studies relating BOLD signals and gamma-band EEG (Logothetis et al., 2001; Foucher et al., 2003; Lachaux et al., 2007). These gamma covariations occurred contralateral to the attended location, consistent with increased neuronal excitability in visual cortical regions that will process the upcoming targets. In contrast, the BOLD signal covaried inversely with occipital alpha-band EEG ipsilateral to the attended direction, representing decreased excitability in visual cortex processing of to-be-ignored locations, but these alpha covariational effects occurred in distinct regions from those observed in the average BOLD and occurred earlier in time than the gammarelated enhancement of to-be-attended locations.

Given that visual placeholders were present throughout the task, it is possible that participants were specifically suppressing the placeholder as a distractor object, akin to the distractor suppression by the pulvinar that has been observed in visual search (Fischer and Whitney, 2012; Strumpf et al., 2013). Cue-elicited anticipatory modulations of alpha-band EEG are still observed in the absence of spatial landmarks (e.g., when suppressing an entire sensory modality; Fu et al., 2001), suggesting that visual landmarks may help one home in on the spatial location to be suppressed (i.e., increase precision), but are not necessary for suppression to occur. Further studies without placeholders would be required to determine whether having a visible object to suppress is necessary to observe this ipsilateral pulvinar activity.

The spatial and time-frequency separation of these effects suggests that attentional suppression and enhancement happen at different points in time in the processing cascade and operate at different levels of visual processing. We suggest that these two attentional mechanisms are deployed in a flexible and adaptive manner based on the demands of the task. In the present study, the entire uncued hemifield was always task irrelevant (i.e., a target would never appear at the uncued location) such that early visual processing for that region of space, or at least from within that visual placeholder, could be suppressed rapidly to limit any distraction from that side. Rather, our task required participants to perform a difficult discrimination of two objects at the cued location, so the effects may have included attentional enhancement specifically in areas necessary for optimal target discrimination performance. Variations in the task demands would likely lead to other patterns of modulation in occipital cortex, with the coordination of suppression and enhancement involving the thalamocortical connections from the pulvinar to the relevant occipital regions. Future studies using multi-methodological approaches will be helpful for further delineation of the subcortical-cortical coordination of attentional control and influence in humans.

\section{References}

Allen PJ, Josephs O, Turner R (2000) A method for removing imaging artifact from continuous EEG recorded during functional MRI. Neuroimage 12:230-239. CrossRef Medline

Boehler CN, Hopf JM, Krebs RM, Stoppel CM, Schoenfeld MA, Heinze HJ, Noesselt T (2011) Task-load-dependent activation of dopaminergic midbrain areas in the absence of reward. J Neurosci 31:4955-4961. CrossRef Medline

Brett M, Anton JL, Valabregue R, Poline JB (2002) Region of interest analysis using an SPM toolbox. Neuroimage 16.

Capotosto P, Babiloni C, Romani GL, Corbetta M (2009) Frontoparietal cortex controls spatial attention through modulation of anticipatory alpha rhythms. J Neurosci 29:5863-5872. CrossRef Medline

Corbetta M, Shulman GL (2002) Control of goal-directed and stimulusdriven attention in the brain. Nat Rev Neurosci 3:201-215. Medline

Doesburg SM, Roggeveen AB, Kitajo K, Ward LM (2008) Large-scale gamma-band phase synchronization and selective attention. Cereb Cortex 18:386-396. CrossRef Medline

Fischer J, Whitney D (2012) Attention gates visual coding in the human pulvinar. Nat Commun 3:1051. CrossRef Medline

Foucher JR, Otzenberger H, Gounot D (2003) The BOLD response and the gamma oscillations respond differently than evoked potentials: an interleaved EEG-fMRI study. BMC Neurosci 4:22. CrossRef Medline

Fu KM, Foxe JJ, Murray MM, Higgins BA, Javitt DC, Schroeder CE (2001) Attention-dependent suppression of distracter visual input can be crossmodally cues as indexed by anticipatory parieto-occipital alpha-band oscillations. Brain Res Cogn Brain Res 12:145-152. CrossRef Medline

Goldman RI, Stern JM, Engel J Jr, Cohen MS (2002) Simultaneous EEG and fMRI of the alpha rhythm. Neuroreport 13:2487-2492. CrossRef Medline

Grandchamp R, Delorme A (2011) Single-trial normalization for eventrelated spectral decomposition reduces sensitivity to noisy trials. Front Psychol 2:236. CrossRef Medline

Harter MR, Miller SL, Price NJ, Lalonde ME, Keyes AL (1989) Neural processes involved in directing attention. J Cogn Neurosci 1:223-237. CrossRef Medline

Hopf JM, Mangun GR (2000) Shifting visual attention in space: an electrophysiological analysis using high spatial resolution mapping. Clin Neurophysiol 111:1241-1257. CrossRef Medline

Huster RJ, Debener S, Eichele T, Herrmann CS (2012) Methods for simultaneous EEG-fMRI: an introductory review. J Neurosci 32:6053-6060. CrossRef Medline

Jensen O, Kaiser J, Lachaux JP (2007) Human gamma-frequency oscillations associated with attention and memory. Trends Neurosci 30:317324. CrossRef Medline

Jung TP, Makeig S, Westerfield M, Townsend J, Courchesne E, Sejnowski T] (2001) Analysis and visualization of single-trial event-related potentials. Hum Brain Mapp 14:166-185. CrossRef Medline

Karnath HO, Himmelbach M, Rorden C (2002) The subcortical anatomy of human spatial neglect: putamen, caudate nucleus and pulvinar. Brain 125:350-360. CrossRef Medline

Kastner S, O'Connor DH, Fukui MM, Fehd HM, Herwig U, Pinsk MA (2004) Functional imaging of the human lateral geniculate nucleus and pulvinar. J Neurophysiol 91:438-448. Medline

Kelly SP, Lalor EC, Reilly RB, Foxe JJ (2006) Increases in alpha oscillatory power reflect an active retinotopic mechanism for distracter suppression during sustained visuospatial attention. J Neurophysiol 95:3844-3851. CrossRef Medline

LaBerge D, Buchsbaum MS (1990) Positron emission tomographic mea- 
surements of pulvinar activity during an attention task. J Neurosci 10: 613-619. Medline

Lachaux JP, Fonlupt P, Kahane P, Minotti L, Hoffmann D, Bertrand O, Baciu M (2007) Relationship between task-related gamma oscillations and BOLD signal: new insights from combined fMRI and intracranial EEG. Hum Brain Mapp 28:1368-1375. CrossRef Medline

Laufs H, Krakow K, Sterzer P, Eger E, Beyerle A, Salek-Haddadi A, Kleinschmidt A (2003) Electroencephalographic signatures of attentional and cognitive default modes in spontaneous brain activity fluctuations at rest. Proc Natl Acad Sci U S A 100:11053-11058. CrossRef Medline

Liu Y, Bengson J, Huang H, Mangun GR, Ding M (2016) Top-down modulation of neural activity in anticipatory visual attention: control mechanisms revealed by simultaneous EEG-fMRI. Cereb Cortex 26:517-529. CrossRef Medline

Liu Z, de Zwart JA, Yao B, van Gelderen P, Kuo LW, Duyn JH (2012) Finding thalamic BOLD correlates to posterior alpha EEG. Neuroimage 63: 1060-1069. CrossRef Medline

Logothetis NK, Pauls J, Augath M, Trinath T, Oeltermann A (2001) Neurophysiological investigation of the basis of the fMRI signal. Nature 412: 150-157. CrossRef Medline

Marshall TR, Bergmann TO, Jensen O (2015) Frontoparietal structural connectivity mediates the top-down control of neuronal synchronization associated with selective attention. PLoS Biol 13:e1002272. CrossRef Medline

Niazy RK, Beckmann CF, Iannetti GD, Brady JM, Smith SM (2005) Removal of FMRI environment artifacts from EEG data using optimal basis sets. Neuroimage 28:720-737. CrossRef Medline

Nunez PL, Srinivasan R (2006) Electric fields of the brain, Ed 2. New York: OUP.

Petersen SE, Robinson DL, Morris JD (1987) Contributions of the pulvinar to visual spatial attention. Neuropsychologia 25:97-105. CrossRef Medline

Posner MI (1980) Orienting of attention. Q J Exp Psychol 32:3-25. CrossRef Medline
Rihs TA, Michel CM, Thut G (2007) Mechanisms of selective inhibition in visual spatial attention are indexed by alpha-band EEG synchronization. Eur J Neurosci 25:603-610. CrossRef Medline

Saalmann YB, Kastner S (2011) Cognitive and perceptual functions of the visual thalamus. Neuron 71:209-223. CrossRef Medline

Saalmann YB, Pinsk MA, Wang L, Li X, Kastner S (2012) The pulvinar regulates information transmission between cortical areas based on attention demands. Science 337:753-756. CrossRef Medline

Scheeringa R, Petersson KM, Kleinschmidt A, Jensen O, Bastiaansen MC (2012) EEG alpha power modulation of fMRI resting-state connectivity. Brain Connect 2:254-264. CrossRef Medline

Shipp S (2004) The brain circuitry of attention. Trends Cogn Sci 8:223-230. CrossRef Medline

Slagter HA, Prinssen S, Reteig LC, Mazaheri A (2016) Facilitation and inhibition in attention: functional dissociation of pre-stimulus alpha activity, P1, and N1 components. Neuroimage 125:25-35. CrossRef Medline

Strumpf H, Mangun GR, Boehler CN, Stoppel C, Schoenfeld MA, Heinze HJ, Hopf JM (2013) The role of the pulvinar in distractor processing and visual search. Hum Brain Mapp 34:1115-1132. CrossRef Medline

Ward LM (2003) Synchronous neural oscillations and cognitive processes. Trends Cogn Sci 7:553-559. CrossRef Medline

Woldorff MG, Liotti M, Seabolt M, Busse L, Lancaster JL, Fox PT (2002) The temporal dynamics of the effects in occipital cortex of visual-spatial selective attention. Brain Res Cogn Brain Res 15:1-15. CrossRef Medline

Woldorff MG, Hazlett CJ, Fichtenholtz HM, Weissman DH, Dale AM, Song AW (2004) Functional parcellation of attentional control regions of the brain. J Cogn Neurosci 16:149-165. CrossRef Medline

Yamagishi N, Goda N, Callan DE, Anderson SJ, Kawato M (2005) Attentional shifts towards an expected visual target alter the level of alpha-band oscillatory activity in the human calcarine cortex. Brain Res Cogn Brain Res 25:799-809. CrossRef Medline 